$\xi=$

\title{
Use of Additive Technologies in Lower Limb Prosthetics
}

\author{
Ivanova E. B ${ }^{1 *}$, Torchinskaya A. ${ }^{2}$ \\ MIREA - Russian Technological University, Moscow, Russia
}

\begin{abstract}
This article is devoted to the development of methods for manufacture of the receiving sleeve of the lower limb prosthesis (PNC) using additive technologies. The technique allows the most accurate transfer of geometry of the patient's stump, increases the speed of its manufacture and provides a non-contact type of interaction with the patient. The proposed development is of interest, as for orthopedic surgeons, and for specialists of companies producing PNK. The use of additive technologies helps to optimize and accelerate the technological process of manufacturing NCP at certain stages, which in turn leads to a decrease in the cost of final products and the time of their production.
\end{abstract}

Keywords: Additive Technologies, lower limb prosthesis, manufacturing

\section{Introduction}

The manufacture of PNK is an important step in the process of medical rehabilitation. Today there is a high need for lower limb prostheses that require maximum personalization and quick replacement.

The modern NCP market includes a significant number of different species. The most popular are modular prostheses. One of the components of the modern modular PNA is a culturereceiving sleeve. The existing technology of its manufacture is costly in time and resources. The rapid development of 3D printing technology allows, if not to the full extent, to partially replace traditional methods.

The use of additive technologies in the manufacture of PNK has many advantages: the possibility for an individual approach to each patient, contactless interaction with the patient, accurate modeling of the technical fit of the caliper, the possibility of manufacturing a culture sleeve with artistic elements [1].

Thus, the development and implementation of the methodology for the manufacture of PNK modules based on additive technologies is an important task.

The youmads are generally classified into three types of biofilms, biochemicals, and biopolymers. Among these materials, bioceramics are of particular importance due to their unique biological properties and their close correspondence with the physiological environment of the body. Biochemicals are the general name of compounds that are produced by imitation of living nature in the laboratory and can be transplanted into living tissues without causing allergy or rejection Biochemicals are metal and non-ferrous materials that are combined with ionic or covalent bonds. These hard and hard materials have poor tensile properties, but have excellent compressive strength, high abrasion resistance and low friction for arched applications. Bio-ceramics, both individually and as biochemical-polymer composites, are the most suitable option for replacing hard and soft tissues among all biomaterials. In terms of biomass, biochemicals have a high rank. Experiences and scientific and technical studies have shown that ceramics are inherently the most biomorphic materials, due to the fact that they are to be found in the nature of ceramic composites compared to other materials - metals and polymers. Most polymers, regardless of poor mechanical properties, are not compatible with the body and do not have a good chemical stability in physiological environments. Although metals have good mechanical properties, they are electrochemically corroded in contact with living tissues of the body. Even the metals that appear to be neutral appear to have undesirable effects in the body, and the fact that most metals, in terms of biocompatibility, are not the right options for use in the body. In the case of ceramics, the story is different. Some of the advantages of ceramics, from a biocompatibility perspective, are:

Generally, ceramics consist of elements that naturally exist in the body, including calcium and phosphorus.

In these combinations, there is no free electron, and therefore, these materials do not often have a weak electrochemical corrosion.

When ceramics are exposed to biological degradation by the body, they can be chemically prolonged for a long time, which can be as long as one human being.

If the body can destroy for some reason bio-thermal, the risk of degradation of ceramics is far less than the risk of metals and polymers in the body.

For the reasons given, it is clear that ceramics are the most compatible and suitable materials for use in the body and in the physiological environment. At present, there is a strong desire to use these materials as implants and biofilm.

\section{Methods and materials}

The proposed technique will allow for the most accurate transfer of the patient's stump geometry, ensuring the best quality of the use of the receiving sleeve, shorten the manufacturing time of the cult-receiving sleeve, provide a non-contact method of interaction with the patient.

The technique is based on the use of information and additive technologies (I \& A).

This process is accompanied by: 
- using new information presentation capabilities - volumetric computer models that transmit individual patient indicators;

- specialized software that allows you to process and edit data for later production;

- technology for accurate prototyping and "conveyor" production of unique products.

Software IAT includes: scanning systems, simulation programs, computer-aided design (CAD) [2]. The advantage of using additive technologies is that all the equipment necessary for the manufacture of the liner takes up little space. In addition, not just "blanks" of the liner are manufactured, but a full-fledged PNK module with mounting seats for calipers and fastening to the body.

The method of manufacturing a culture receiving sleeve includes several steps (Figure 1):

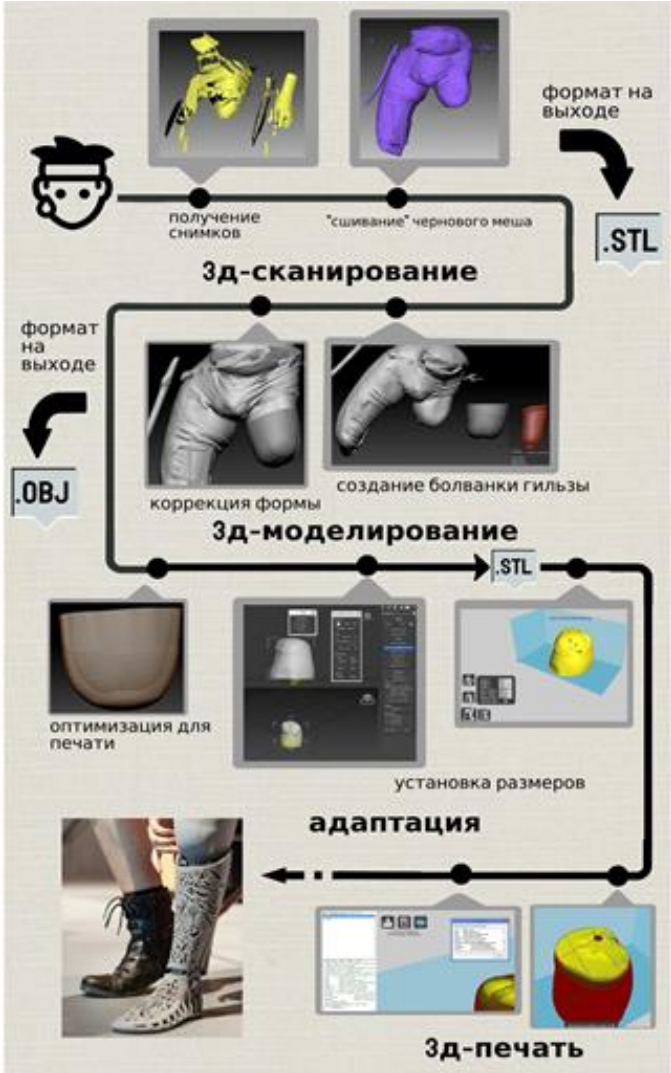

Figure 1: Diagram of the technique of applying 3D printing technology in lower limb prosthetics

- Selection of the type of prosthesis, consultation with an orthopedic surgeon.

- 3D scanning of a patient's stump is the process of obtaining data on the geometry of a necessary object, suitable for the subsequent creation of a mathematical three-dimensional model of this object, using special equipment - 3D scanners [3].

- 3D modeling - the transformation process obtained after 3D scanning of the Mesh object, while preserving the geometry, creating seats for fixing the remaining modules of the prosthesis, editing and shaping the sleeve of any desired shape.

- Adaptation of 3D models for printing.

- 3D printing or "additive production" - the process of creating solid three-dimensional objects of almost any geometric shape based on a digital model [4].

$3 \mathrm{D}$ printing is based on the concept of constructing an object in succession applied layers that display the outlines of the model. In fact, 3D printing is the exact opposite of traditional methods of mechanical production and processing, such as milling or cutting, where the appearance of the product is formed by removing excess material (the so-called "subtractive production").

Upon completion we get the finished cult-receiving sleeve.
Each stage of the implementation of the methodology requires the use of specialized software (software) and equipment.

Therefore, for testing the proposed methodology, the following configuration of software and hardware was selected, table 1 .

Table 1: Completion of software and hardware

\begin{tabular}{|c|c|c|}
\hline Stage & Hardware $\backslash$ Software & Properties $\backslash$ Method \\
\hline 3D scanning & ArtecEva & Contactless scan method \\
\hline $\begin{array}{c}\text { Point cloud } \\
\text { processing }\end{array}$ & ArtecStudio 9 & Accompanying software \\
\hline 3D modeling & AutodeskFUSION 360 & $\begin{array}{c}\text { Parametric and solid } \\
\text { modeling }\end{array}$ \\
\hline $\begin{array}{c}\text { Preparing for } \\
\text { 3D printing }\end{array}$ & CURA 2.1 & Free open source software \\
\hline 3D printing & PrusaI3 Hephestos & Fdm \\
\hline Material & Filamentarno abs & ABS \\
\hline
\end{tabular}

The practical implementation of the method of manufacturing a model of a cultured receiving sleeve for a child NCP began with the construction of surface and solid-state modeling with the use of model methods based on a previously obtained stump model using 3D scanning (Figure 2).

Studies have shown the need to connect polygonal and solid modeling by introducing new software and optimizing production compliance.

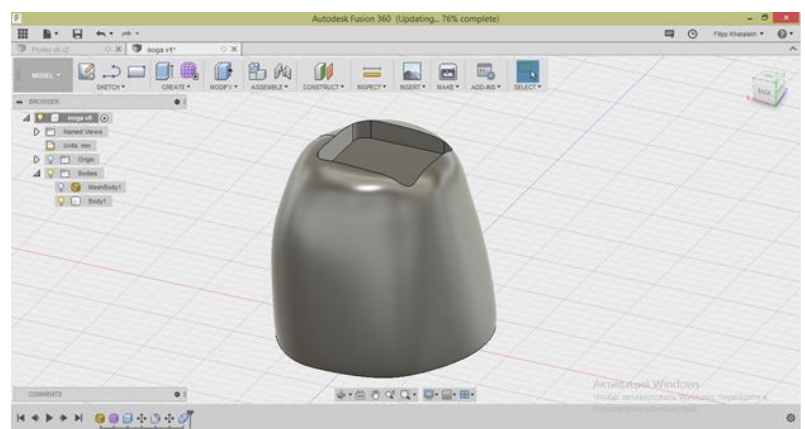

Figure 2: Ready-made model of a cult-receiving sleeve in solid-state form with a seat for the RSU platform

Further, the cultivation of the layout of the cult-receiver sleeve of the children's NCP was performed on a rapid prototyping unit (Figure

$3)$.

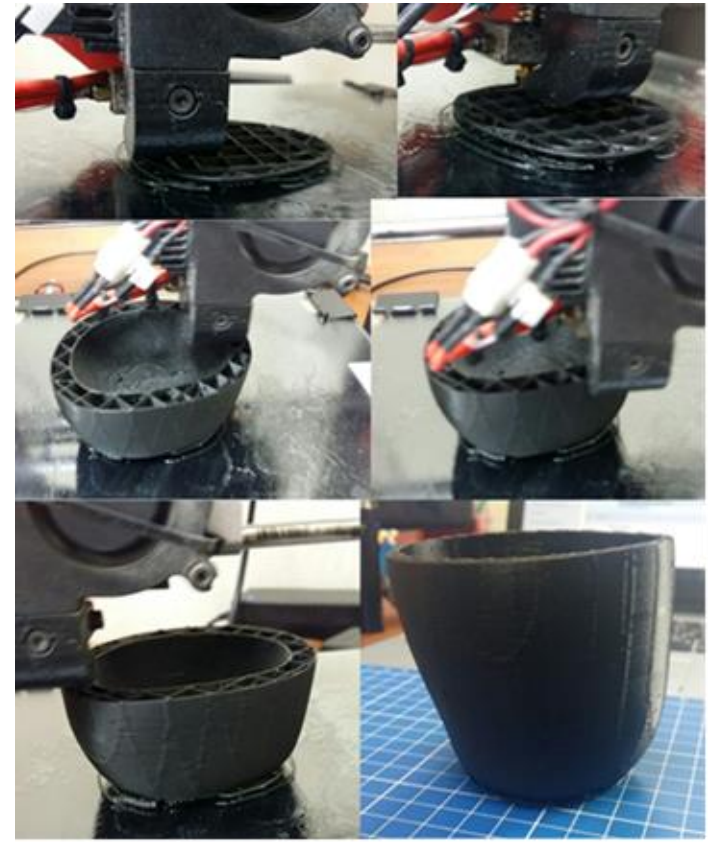

Figure 3: Stages of 3D printing of a culture receiving sleeve 


\section{Results and discussion}

In the process of performing this work, when constructing a 3D model, solid-state modeling methods were used, instead of the polygonal modeling methods which were used earlier. As a result, the surface quality and topology of the model was improved. It is obvious that solid modeling does not have such "flexibility" as a polygonal one, but it has a number of advantages in terms of manufacturability.

For example, "wiping" the bottom of the sleeve gives a large area of contact between the silicone lining of the prosthesis (liner), which increases the prodility of the prosthesis and prolongs the life of the liner (Figure 4).

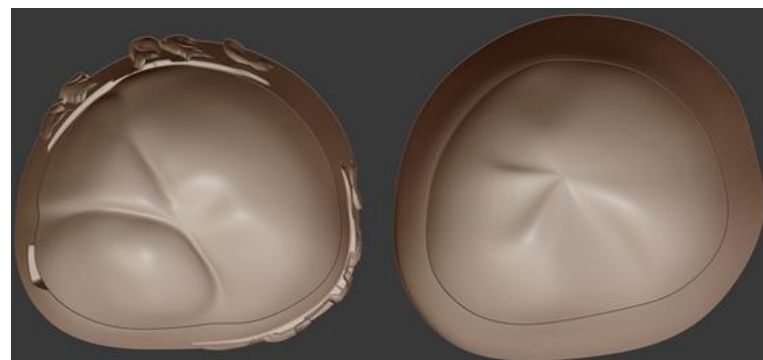

Figure 4: Section of 3D models of cult-of-receiving sleeves (polygonal model - on the left) and (solid model - right)

Practically it has been established that, at the time, the entire technological chain (Figure 1) fits into one working day and is performed by one specialist on one PC.

\section{Conclusion}

The proposed technique fully utilizes the capabilities of additive technologies to create lower-limb prosthetic cultured receiving sleeves and will increase the level of personalization, satisfy artistic needs [5], significantly reduce production time from data acquisition to finished products, localize all equipment on one small platform and reduce a staff of up to one specialist. Its use in the production of necessary products in series of various scales will undoubtedly increase to quality of medical care.

\section{References}

[1] Jan Larsen. Additive and hybrid production using 3D printing. // CAD / CAM / CAE Observer. - № 3 (95), 2015, C. 56-58

[2] Valery Kukushkin, Alexander Kuznetsov. JSC Stankoprom is a system integrator in the development of additive technologies. // Stankoinstrument. - № 1, 2016, pp. 46 - 53

[3] Professional 3D handheld scanners. [Electronic resource] http://habrahabr.ru/post/232387/ - (appeal date 07/24/2016).

[4] Makarov M. Industrial 3D-printers. // Photonics. - № 5, 2015, C. 53

[5] Sokolova M.L. Modern problems of the direction "Technology of artistic processing of materials." // Russian technological journal. №5 (1), 2017, p. 50-56. 